\title{
Review of: "Biochemical and Cytological Interactions Between Callose Synthase and Microtubules in the Tobacco Pollen Tube"
}

Aslihan Cetinbas Genc ${ }^{1}$

1 Marmara University

Potential competing interests: The author(s) declared that no potential competing interests exist.

I think it is a guiding article for researchers working on the subject will refer to. I congratulate the authors for writing a very descriptive article on a subject with limited literature. 